\title{
Ab initio Evaluation of Intramolecular Electron Transfer Reactions in Halobenzene Radical Anions and Stabilized Derivatives
}

\author{
Adriana B. Pierini* and D. Mariano A. Vera \\ INFIQC. Departamento de Química Orgánica - Facultad de Ciencias Químicas - Universidad \\ Nacional de Córdoba. \\ Ciudad Universitaria - 5000 Córdoba, Argentina
}

Supplementary Material

Contents

PART 1. The main properties for the most stable $\pi$ and $\sigma$ type structures calculated by means of different formalisms. Properties obtained from the PES analysis compared to the experimental values where available

Table S1. RAs of $\mathrm{PhCl}$ calculated by different formalisms ................................ S3

Table S2 a. PES Analysis for the haloabenzenes ….......................................... S4

Table S2 b. PES Analysis for PhCl. Comparisson of results obtained at different levels of theory

Figure S1.

PART 2. PES profiles obtained for $\mathrm{PhCl}, \mathrm{PhBr}$ and $\mathrm{PhI}$ at different levels of theory

Figure S2

Figure S3

Figure S4

Figure S5 S9 
Part I. The main properties for the most stable $\pi$ and $\sigma$ type structures calculated by means of different formalisms. Properties obtained from the PES analysis compared to the experimental values where available. 
Table S1 . RAs of $\mathrm{PhCl}$ calculated by different formalisms.

\begin{tabular}{|c|c|c|c|c|c|c|c|c|c|c|}
\hline \multirow[t]{2}{*}{ Formalism } & \multirow[t]{2}{*}{ State } & \multirow[t]{2}{*}{ RA } & \multirow[t]{2}{*}{ symm. } & \multirow[t]{2}{*}{$E^{(a)} /$ Hartee } & \multirow[t]{2}{*}{$r(\mathrm{CCl}) / \AA$} & \multicolumn{3}{|c|}{$r(\mathrm{CC}) / \AA^{(b)}$} & \multirow[t]{2}{*}{$d(\mathrm{Cl}<\mathrm{Ph})^{(\mathrm{c})}$} & \multirow[t]{2}{*}{$q(\mathrm{Cl})^{(d)}$} \\
\hline & & & & & & $i-O$ & $o-m$ & $m-p$ & & \\
\hline \multirow[t]{2}{*}{$\mathrm{UHF} / 6-31+\mathrm{G}^{*}$} & ${ }^{2} \mathrm{~A}_{2}$ & $\pi$ & $C_{2 v}$ & -689.54464 & 1.788 & 1.38 & 1.46 & 1.39 & 180 & -0.075 \\
\hline & ${ }^{2} \mathrm{~A}_{1}$ & $\sigma$ & & Repulsive $^{(f)}$ & & & & & & \\
\hline \multirow[t]{2}{*}{ UB3LYP/3-21G* } & ${ }^{2} \mathrm{~A}_{2}$ & $\pi$ & $C_{2 v}$ & -688.42672 & 1.838 & 1.39 & 1.47 & 1.40 & 180.0 & -0.174 \\
\hline & ${ }^{2} \mathrm{~A}_{1}$ & $\sigma$ & $C_{2 v}$ & -688.46730 & 2.655 & 1.39 & 1.40 & 1.40 & 180.0 & -0.729 \\
\hline \multirow[t]{2}{*}{ UB3LYP/6-31G* } & ${ }^{2} \mathrm{~A}_{2}$ & $\pi$ & $C_{2 v}$ & -691.78821 & 1.826 & 1.39 & 1.46 & 1.40 & 180.0 & -0.218 \\
\hline & ${ }^{2} \mathrm{~A}_{1}$ & $\sigma$ & $C_{2 v}$ & -691.82714 & 2.698 & 1.39 & 1.40 & 1.40 & 179.9 & -0.757 \\
\hline \multirow[t]{2}{*}{ UB3LYP/LANL2DZ // UB3LYP/3-21G* } & ${ }^{2} \mathrm{~A}_{2}$ & $\pi$ & $C_{2 v}$ & -246.50880 & & & & & & -0.236 \\
\hline & ${ }^{2} \mathrm{~A}_{1}$ & $\sigma$ & $C_{2 v}$ & -246.54371 & & & & & & -0.670 \\
\hline \multirow[t]{2}{*}{ UB3LYP / 6-31+G* } & ${ }^{2} \mathrm{~A}_{2}$ & $\pi$ & $C_{2 v}$ & -691.82152 & 1.81 & 1.39 & 1.46 & 1.40 & 180.0 & -0.071 \\
\hline & ${ }^{2} \mathrm{~A}_{1}$ & $\sigma$ & $C_{2 v}$ & -691.85424 & 2.743 & 1.39 & 1.41 & 1.40 & 179.9 & -0.742 \\
\hline \multirow[t]{2}{*}{ PMP2/6-31+G* } & ${ }^{2} \mathrm{~A}_{2}$ & $\pi$ & $C_{2 v}$ & -690.45312 & 1.788 & 1.39 & 1.45 & 1.39 & 180.0 & -0.069 \\
\hline & ${ }^{2} \mathrm{~A}_{1}$ & $\sigma$ & $C_{2 v}$ & -690.46829 & 2.635 & 1.36 & 1.38 & 1.38 & 180.0 & -0.894 \\
\hline \multirow[t]{2}{*}{ UB3LYP/6-311+G(2d,p) //UB3LYP/6-31G* } & ${ }^{2} \mathrm{~A}_{2}$ & $\pi$ & $C_{2 v}$ & -691.91131 & 1.826 & 1.39 & 1.46 & 1.40 & 180.0 & -0.214 \\
\hline & ${ }^{2} \mathrm{~A}_{1}$ & $\sigma$ & $C_{2 v}$ & -691.94078 & 2.698 & 1.39 & 1.40 & 1.40 & 180.0 & -0.758 \\
\hline \multirow[t]{2}{*}{$\mathrm{UCCSD} / 6-31+\mathrm{G}^{*} / / \mathrm{UCCD} / 6-31 \mathrm{G}^{*}$} & ${ }^{2} \mathrm{~A}_{2}$ & $\pi$ & $C_{2 v}$ & -690.49280 & 1.795 & 1.39 & 1.46 & 1.39 & 180.0 & -0.077 \\
\hline & ${ }^{2} \mathrm{~A}_{1}$ & $\sigma$ & $C_{2 v}$ & -690.51855 & 2.721 & 1.38 & 1.38 & 1.37 & 180.0 & -0.914 \\
\hline \multirow[t]{2}{*}{$\mathrm{UCCSD}(\mathrm{T}) / 6-31+\mathrm{G}^{*} / / \mathrm{UCCD} / 6-31 \mathrm{G}^{*}$} & ${ }^{2} \mathrm{~A}_{2}$ & $\pi$ & $C_{2 v}$ & -690.53525 & & & & & & -0.077 \\
\hline & ${ }^{2} \mathrm{~A}_{1}$ & $\sigma$ & $C_{2 v}$ & -690.55928 & & & & & & -0.916 \\
\hline
\end{tabular}

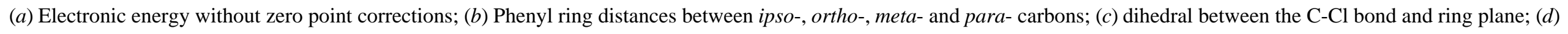

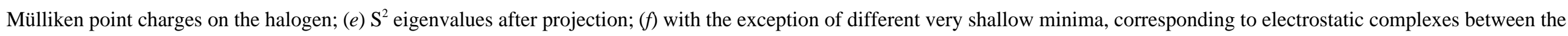
halide and radical beyond $3.5 \AA$. 
Table S2 a) PES Analysis for the haloabenzenes ${ }^{(a, b)}$

\begin{tabular}{|c|c|c|c|c|c|c|c|c|}
\hline \multirow[t]{2}{*}{ species } & \multicolumn{2}{|c|}{$\mathrm{PhF}$} & \multicolumn{2}{|c|}{$\mathrm{PhCl}$} & \multicolumn{2}{|c|}{$\mathrm{PhBr}$} & \multicolumn{2}{|c|}{$\mathrm{PhI}$} \\
\hline & calculated & experimental & calculated & experimental & calculated & experimental & calculated & experimental \\
\hline$E_{v}\left(\pi^{*}\right),(\mathrm{PhF}, \mathrm{PhCl}, \mathrm{PhBr}) ; E_{v}\left(\sigma^{*}\right),(\mathrm{PhI})^{(c)}$ & 25.93 & 20.06 & 23.04 & 17.3 & 21.92 & 16.1 & 23.78 & \\
\hline$E A(\mathrm{X})^{(d)}$ & 80.28 & & 85.76 & 83.48 & 82.91 & 77.6 & 68.71 & 69.41 \\
\hline$\Delta E_{\sigma \pi}^{(e)}$ & 10.08 & & -18.49 & & -26.28 & & -36.37 & \\
\hline$D(\mathrm{Ph}-\mathrm{X})^{(f)}$ & 125.27 & 125.00 & 92.30 & 92.01 & 79.8 & 80.5 & 63.9 & 65.45 \\
\hline$E^{\#}(\pi-\sigma)^{(g)}$ & 19.90 & & 1.00 & & $\sim 0.0$ & & earlier & \\
\hline$E^{\sharp}\left(\mathrm{PhX}+\mathrm{e} \rightarrow \mathrm{Ph} \cdot+\mathrm{X}^{-}\right) ;[$experimental $\left.\sigma] \mathrm{h}^{g}\right)$ & 66.15 & & 21.05 & {$[1.4]$} & 19.5 & {$[9.3]$} & 9.97 & {$[640]$} \\
\hline$\Delta E\left(\mathrm{PhX}+\mathrm{e} \rightarrow \mathrm{Ph} \cdot+\mathrm{X}^{-}\right)^{(i)}$ & 66.15 & & 16.70 & 12.5 & 4 & 3.22 & -4.8 & -5.53 \\
\hline
\end{tabular}

(a) All energies in kcal/mol.

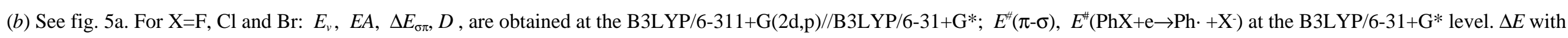
B3LYP/LANL2DZ basis. For PhI, all parameters with LANL2DZ basis.

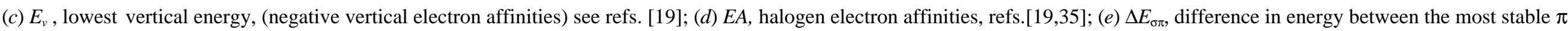

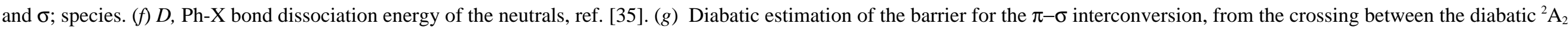

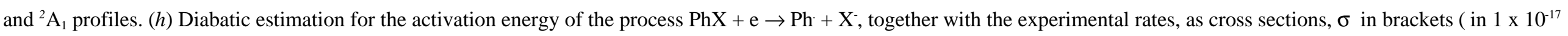
molecules $\mathrm{cm}^{-2}$ ); see ref. [36]. (i) Exothermicity of the process $\mathrm{PhX}+\mathrm{e} \rightarrow \mathrm{Ph}+\mathrm{X}$ from thermal dissociative electron attachment experiments; see ref. [36]. 
Table S2 b) PES Analysis for chlorobenzene. Comparisson of results obtained at different levels of theory ${ }^{(a)}$

\begin{tabular}{|c|c|c|c|c|c|c|c|c|}
\hline & $\begin{array}{l}\text { B3LYP } \\
\text { /6-31G* }\end{array}$ & $\begin{array}{c}\text { B3LYP } \\
\text { /LANL2DZ }\end{array}$ & $\begin{array}{l}\text { B3LYP } \\
/ 6-31+G^{*}\end{array}$ & $\begin{array}{c}\text { B3LYP/6-311+G(2d,p) } \\
\text { // B3LYP/6-31+G* }\end{array}$ & $\begin{array}{c}\text { PMP2 } \\
/ 6-31+G^{*}\end{array}$ & $\begin{array}{l}\text { CCSD/6-31+G* } \\
/ / C C D / 6-31 G^{*}\end{array}$ & $\begin{array}{c}\operatorname{CCSD}(\mathrm{T}) / 6-31+\mathrm{G}^{*} \\
/ / \mathrm{CCD} / 6-31 \mathrm{G}^{*}\end{array}$ & experimental \\
\hline$E_{v}\left(\pi^{*}\right)$ & 40.98 & 33.70 & 25.10 & 23.04 & 33.52 & 38.08 & 33.67 & 17.3 \\
\hline$E A(\mathrm{Cl})$ & 72.77 & 64.36 & 85.61 & 85.76 & 72.75 & 70.72 & & 83.48 \\
\hline$\Delta E_{\sigma \pi}$ & -24.49 & -21.91 & -20.61 & -18.49 & -9.52 & -16.16 & -15.08 & \\
\hline$D(\mathrm{Ph}-\mathrm{Cl})$ & 92.52 & 81.07 & 90.78 & 92.30 & 100.78 & 91.99 & 95.07 & 92.01 \\
\hline$E^{\#}(\pi-\sigma)^{(k)}$ & 2.18 & & 1.00 & & 7.40 & & & \\
\hline$E^{\#}(\mathrm{PhCl}+\mathrm{e} \rightarrow \mathrm{Ph} \cdot+\mathrm{Cl}) ;[$ experimental $\sigma]$ & 21.05 & & 14.17 & & & & & {$[1.4]$} \\
\hline$\Delta E(\mathrm{PhCl}+\mathrm{e} \rightarrow \mathrm{Ph} \cdot+\mathrm{Cl})$ & 19.74 & 16.71 & 5.16 & 6.55 & 28.03 & 21.27 & 23.51 & 12.5 \\
\hline
\end{tabular}

(a) See Figure 5a; all defined as in Table 1. 


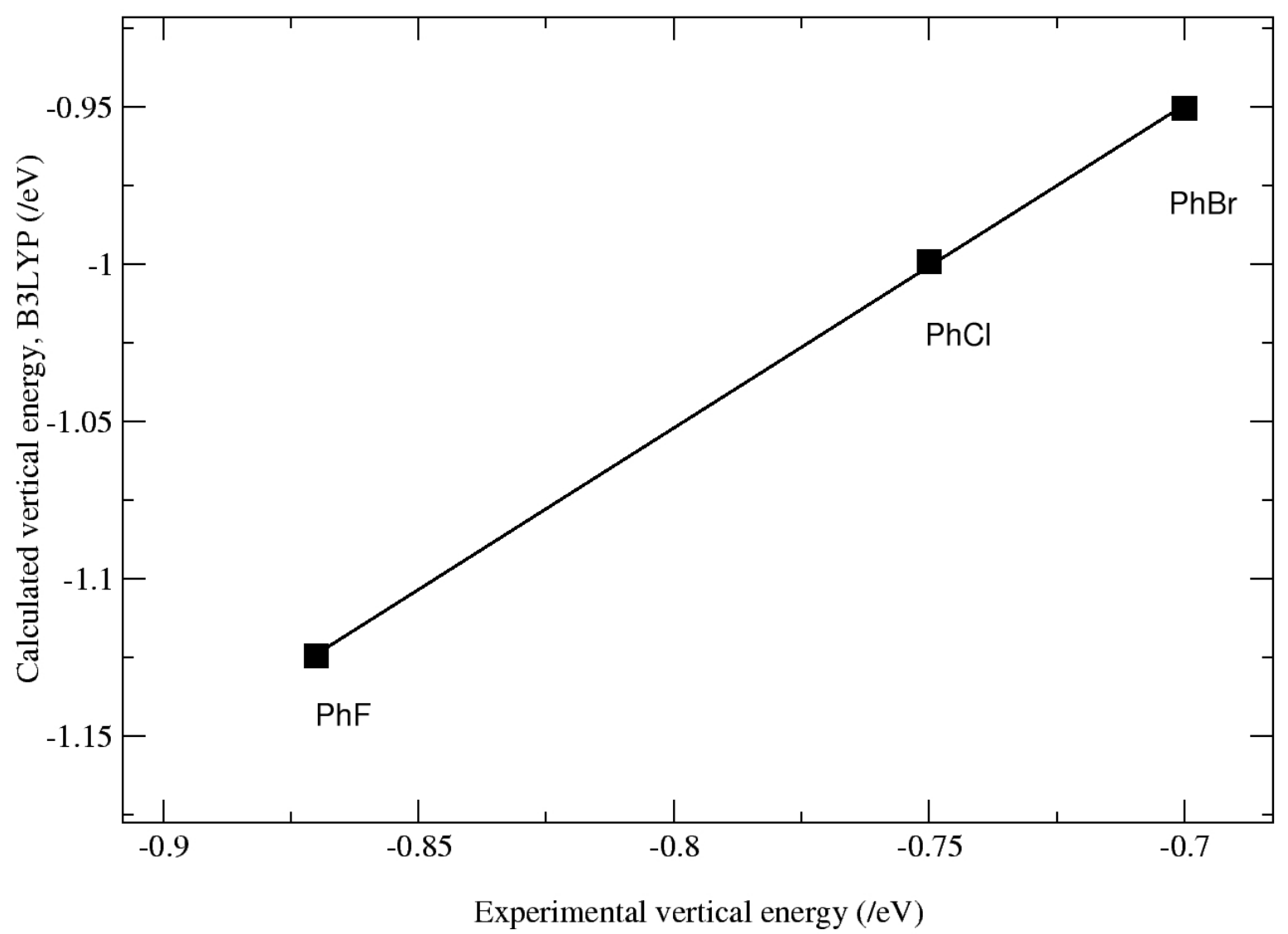

FIGURE S1. The absolute values at $6-311+\mathrm{G}(2 \mathrm{~d}, \mathrm{p})$ have some overestimation of the order of $0.2 \mathrm{eV}$, the relative values can be reproduced within $0.015 \mathrm{eV}$. Recently, Wetmore et al. (see ref. [20c]) have accounted for the right first vertical energy of uracil and thymine with an absolute error smaller than $0.04 \mathrm{eV}$ using the same functional and the $6-311+\mathrm{G}(2 \mathrm{~d} f, \mathrm{p})$ basis. As well as for halobenzenes, these compounds have negative electron affinities. Recently, also Schaeffer (see. Rienstra-Kiracofe, J. C.; Barden, C. J; Brown, T; Schaefer III, H. F., J. Phys.

Chem. A, 2001, 105, 524-528) accounted for the negative thermal electron affinity of the naphtalene within 0.01 $\mathrm{eV}$. 

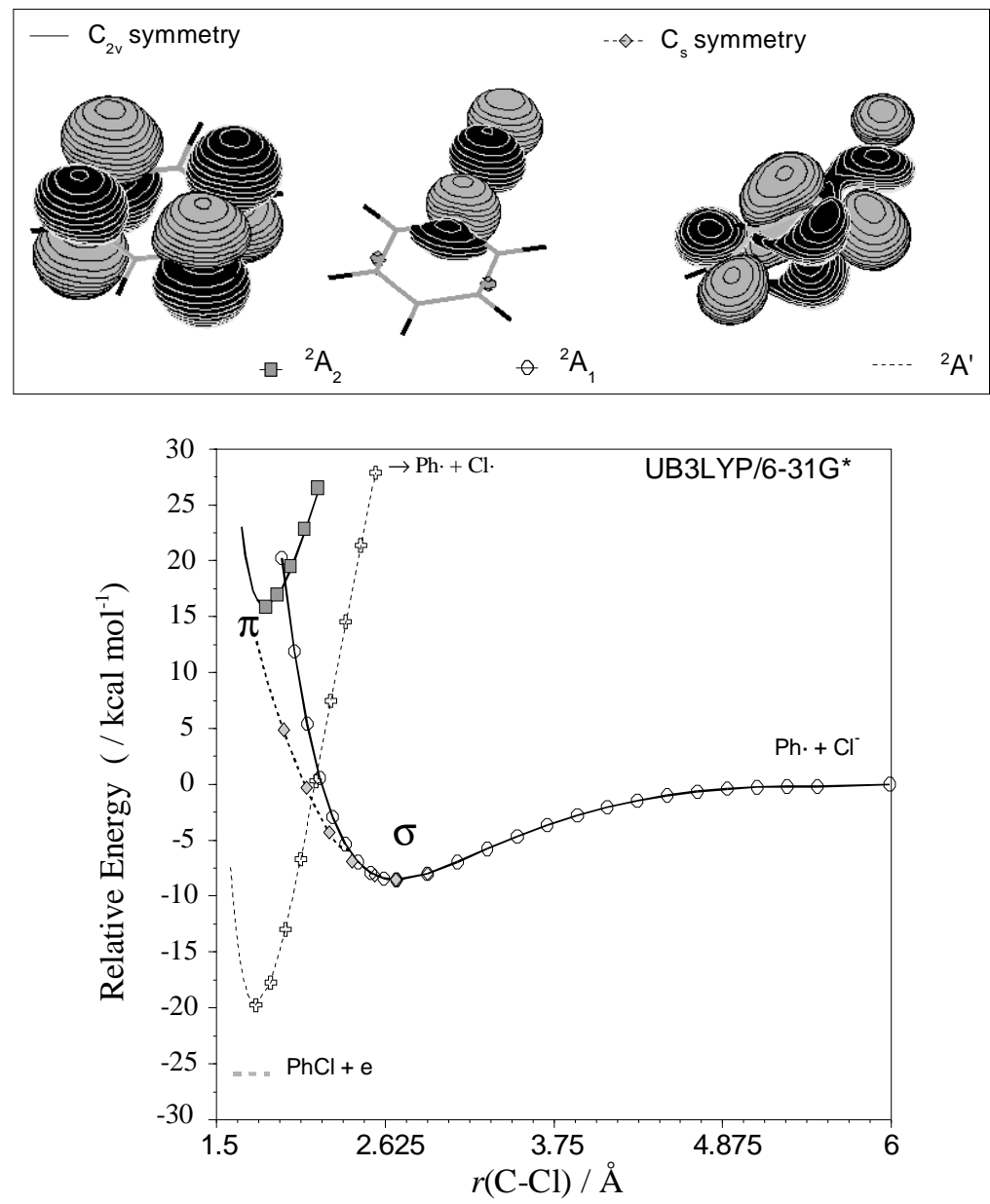

FIGURE S2. (a) Main PES profiles obtained for $\mathrm{PhCl}$ an its anionic surfaces. The neutral molecule plus an electron ( $\cdots$ ), the diabatic $\pi$ and $\sigma$ curves (-), and the low symmetry path, where the $\pi-\sigma$ coupling is allowed by the loss of the planarity (---). The SOMOs symmetry is indicated in the above orbital diagrams.

(b) Comparison of the $\pi-\sigma$ crossing zone for the $\mathrm{RAs}$ of $\mathrm{PhF}$ and $\mathrm{PhCl}$ (see text). 


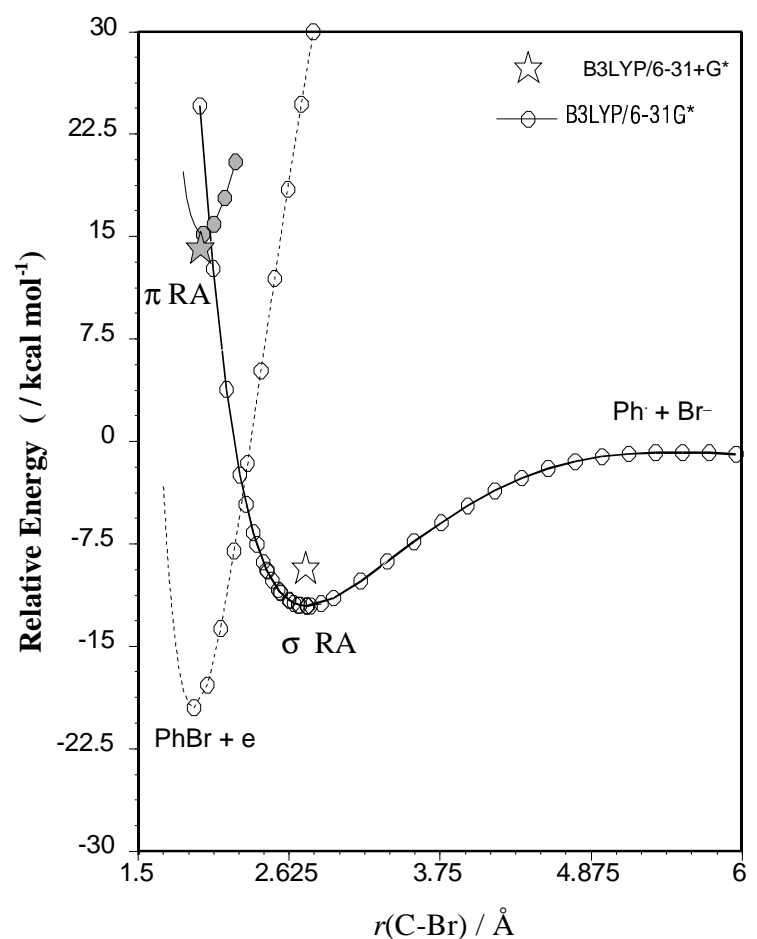

FIGURE S3. Neutral and anionic profiles obtained for $\mathrm{PhBr}$.

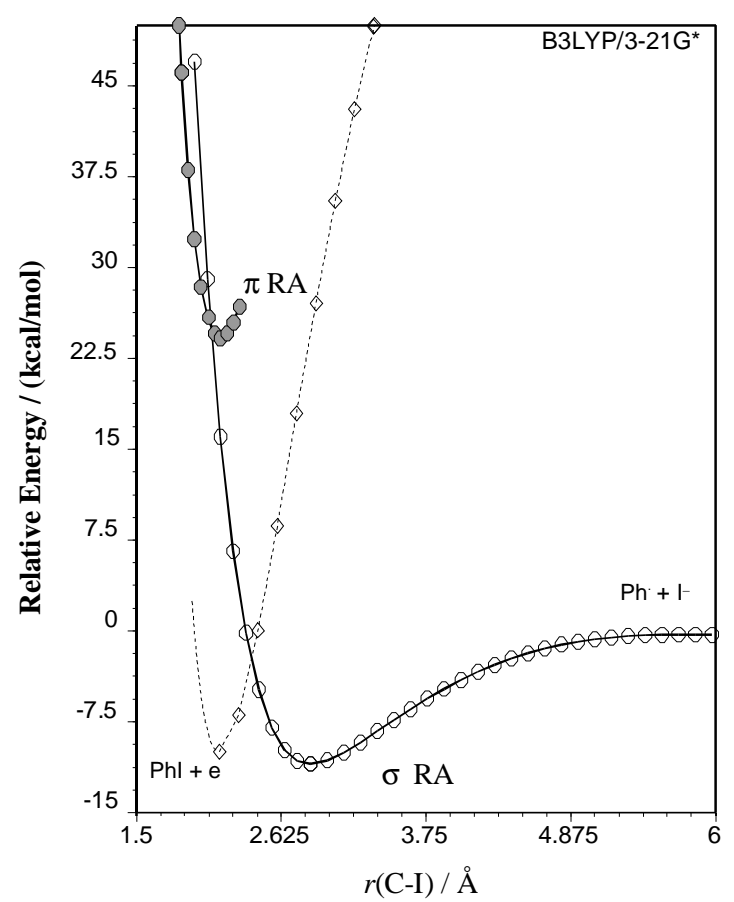

FIGURE S4. Neutral and anionic profiles obtained for PhI. 


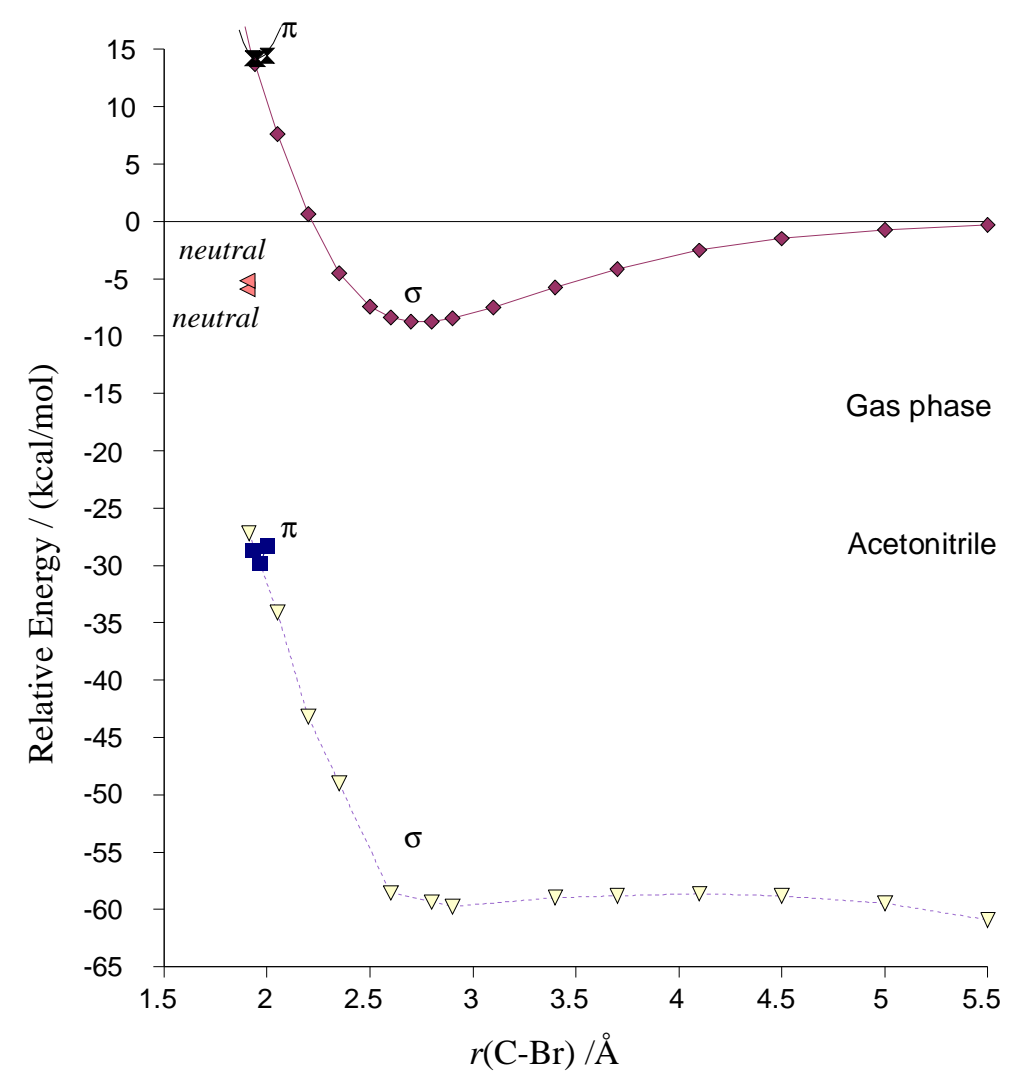

FIGURE S5. PhBr fragmentation profile in gas phase and acetonitrile (B3LYP/6-31+G*). 